\title{
Médiévales
}

Langues, Textes, Histoire

49 | automne 2005

La paroisse, genèse d'une forme territoriale

\section{L'horlogerie et la mécanique de l'allégorie chez Jean Froissart}

Clockmaking and the Mechanics of Allegory in Jean Froissart's

Julie Singer

\section{OpenEdition}

1 Journals

Édition électronique

URL : https://journals.openedition.org/medievales/1254

DOI : 10.4000/medievales. 1254

ISSN : $1777-5892$

Éditeur

Presses universitaires de Vincennes

Édition imprimée

Date de publication : 1 décembre 2005

Pagination : 155-172

ISBN : 2-84292-177-1

ISSN : 0751-2708

Référence électronique

Julie Singer, «L'horlogerie et la mécanique de l'allégorie chez Jean Froissart », Médiévales [En ligne], 49 | automne 2005, mis en ligne le 05 mars 2008, consulté le 22 avril 2022. URL : http://

journals.openedition.org/medievales/1254; DOI : https://doi.org/10.4000/medievales.1254

Ce document a été généré automatiquement le 22 avril 2022.

Tous droits réservés 


\title{
L'horlogerie et la mécanique de l'allégorie chez Jean Froissart ${ }^{1}$
}

\author{
Clockmaking and the Mechanics of Allegory in Jean Froissart's
}

\section{Julie Singer}

1 L'Orloge amoureus de Jean Froissart, un poème narratif rédigé vers 1368, consiste en une description allégorique de la psychologie de l'amant, construite à partir d'une comparaison : l'amant est comme une horloge. Cet emploi de l'horloge comme point de départ d'une allégorie amoureuse a été durement critiqué par Johan Huizinga dans L'Automne du Moyen Âge. «Le symbolisme était usé, » dit-il. « La recherche de symboles et d'allégories était devenue un vain jeu d'esprit, une fantaisie superficielle sur une seule analogie... dès que la manie du symbolisme s'applique aux matières profanes ou simplement morales, la décadence apparaît ${ }^{2}$. Le premier exemple qu'il fournit de cette « décadence » est, bien sûr, l'Orloge amoureus. Mais loin d'être un texte que nous pouvons rejeter comme «décadent ", le poème de Froissart a le mérite de témoigner d'une étape importante dans l'histoire de l'horlogerie, d'engager une réflexion sur l'importance du nombre et de la mesure dans la poésie et dans le comportement humain et de nous divertir. Je propose donc de lire le texte en prêtant une attention particulière aux enjeux mathématiques, technologiques et allégoriques implicites dans l'usage de la figure de l'horloge. Après un résumé de l'histoire de l'horlogerie au XIv siècle, il conviendra de situer l'orloge amoureus dans le contexte d'autres ouvrages contemporains traitant de l'horloge ou de l'horlogerie.

L'horlogerie au XIV siècle

2 L'horloge mécanique telle que Froissart la décrit est une innovation technique du XIV siècle, dont l'un des exemples les plus connus aujourd'hui est l'horloge sonnante que Charles V fit installer au palais royal sur l'île de la Cité. Cette horloge, construite par l'horloger allemand Henri de Vic entre 1362 et 1370, eut un tel succès que le roi fit installer d'autres horloges au Château de Vincennes et à l'Hôtel Saint-Pol. Jean Golein écrit dans sa traduction du Rationale divinorum officiorum que le roi ordonna aux églises de Paris de faire sonner leurs cloches en même temps que son horloge pour que tous les Parisiens sachent l'heure : "Car luise le soleil ou non l'on scet touz jours les heures 
sanz defaillir par ycelles cloches bien attrampes ${ }^{3}$. Les historiens ont tendance à interpréter ce décret comme un moment charnière dans le passage du temps liturgique, caractérisé par l'inégalité des heures, au temps moderne, qui divise le jour en vingt-quatre heures égales. Jacques Le Goff distingue entre ce qu'il appelle le «temps de l'église » et le «temps du marchand ${ }^{4}$ : l'émergence de l'heure fixe aurait convenu à la bourgeoisie et changé la manière dont les ouvriers étaient payés. Le décret de Charles V, qui avait pour but de régulariser le passage des heures à Paris, serait donc un événement d'une importance capitale dans l'histoire économique et culturelle de la France. Cependant, comme la remarque de Jean Golein est la seule référence contemporaine au décret connue des chercheurs modernes, Gerard Dohrn-Van Rossum met en doute l'idée que Charles $\mathrm{V}$ ait jamais fait une telle loi ${ }^{5}$. Même dans sa discussion des habitudes quotidiennes de "nostre roy bien ordenné " ${ }^{6}$, dans lequel elle raconte l'histoire du roi Elfredes d'Angleterre et de sa chandelle divisée en vingt-quatre parties, Christine de Pizan ne mentionne jamais l'horloge du palais.

3 En tout cas, quelle que soit sa signification dans l'histoire du travail, l'importance de l'horloge du palais royal dans l'œuvre de Froissart est claire : Peter Dembowski nous dit dans la préface de son édition de l'Orloge que Froissart aurait eu l'occasion de voir l'horloge et de parler avec Henri de Vic avant sa réalisation en 1370, et que «les historiens de l'horlogerie associent le poème... avec l'horloge du palais " ${ }^{7}$. Même si rien ne permet d'affirmer que le poème fait explicitement référence à l'horloge du palais, il est clair que Froissart avait des connaissances en horlogerie mécanique.

4 L'Orloge amoureus est un poème de 1174 vers, conservé dans un seul manuscrit (BnF fr. 830), qui raconte l'état d'âme de l'amant en s'appuyant à la fois sur les personnifications amoureuses familières dès le Roman de la Rose et sur l'horloge mécanique. Malgré sa vision assez conventionnelle de l'amour, résumer le texte est difficile en raison de l'absence d'action- Michel Zink décrit, non sans ironie, le caractère statique et atemporel du poème - et de sa structure tripartite. Après une brève introduction, le texte prend la forme de trois « modes » discursifs simultanés que j'appellerai «mode technique », «mode allégorique » et «mode direct ». Les passages écrits sur le mode technique, copiés à l'encre rouge dans le manuscrit, traitent du fonctionnement mécanique de l'horloge. Les parties écrites sur le mode allégorique établissent les similitudes entre les différentes parties de l'horloge et des objets ou des qualités, en utilisant le langage courant des personnifications courtoises (par exemple, le dyal représente Dous Pensier). Le mode direct est constitué d'une adresse directe à la dame aimée. Ces trois modes alternent, dans ce même ordre, tout au long du texte.

5 Le poème commence avec une identification qui prend quasiment le caractère d'une équation mathématique.

" Je me puis bien comparer a l'orloge,

Car quant Amours, qui en mon coer se loge,

M'i fait penser et mettre y mon estude,

G'i aperçoi une similitude. » (vv. 1-4)

6 Le système représentatif du poème se fonde sur cette identification entre l'amant et l'horloge. Avant de procéder à une interprétation du poème, il convient de s'interroger sur la nature de l'horloge à la fin du XIV ${ }^{\mathrm{e}}$ siècle.

7 Tout d'abord, le mot horloge et ses variantes médiévales - oriloge, ologe, et cætera sont des néologismes, et jusqu'au dix-neuvième siècle, le mot horloge a la particularité de pouvoir être masculin ou féminin, une particularité qui survit, à titre d'exemple, 
dans le nom du Gros Horloge de Rouen. Froissart l'emploie au masculin, genre qui facilite son assimilation au corps de l'homme, souligne son identification avec le cœur masculin et consolide son rôle dans la représentation de l'état d'âme d'un amant.

Avant la fin du treizième siècle, la plupart des villes et monastères dépendaient de cadrans solaires ou de clepsydres (horloges hydrauliques), ce qui posait un problème quand le soleil ne brillait pas ou quand l'eau gelait en hiver. L'innovation technique qui permet le développement de l'horloge mécanique est l'échappement à roue de rencontre, qui s'articule avec le mécanisme régulateur appelé le foliot. Bien que certains croient voir ces structures attestées plus tôt - dans un dessin de Villard de Honnecourt notamment-, rien ne nous permet de placer l'invention de l'horloge mécanique avant les dernières années du XIII ${ }^{e}$ siècle. Les premières attestées datent du début $\mathrm{du} \mathrm{XIV}^{\mathrm{e}}$ siècle. Elles furent installées à Milan, à Padoue, à Pavie, à Bruges, à Strasbourg. La vogue de l'horloge dans les années 1360-1370 s'est manifestée par une véritable explosion du nombre des villes qui en possèdent.

Très peu ont survécu - les plus anciennes qui sont toujours en état de marche sont celles de Salisbury et de Wells, dit-on. Mais notre connaissance de ces machines est étayée par quelques textes de l'horlogerie du XIV siècle qui nous sont parvenus: le Tractatus Astrarii de Giovanni de'Dondi (1364) et un traité anonyme en français. La très célèbre horloge astronomique de Dondi était une structure heptagonale avec une face qui montrait le mouvement de chacune des "planètes » connues, ainsi qu'une horloge proprement dite et un calendrier des fêtes mobiles. Philippe de Mézières, dans son Songe du vieil pélerin, exprime son admiration pour l'Astrario du maître italien :

«Entre lesquelles œuvres il a fait un grant instrument, par aucuns appelle l'espere ou orloge du mouvement du ciel. Ouquel instrument sont tous les meuvemens des signes [et] des planetes, avecques leurs cercles et epicycles et differences, par multiplication de roes sans nombre, avecques toutes leurs parties; et a chacune planete, en ladicte espere, est particulierement son mouvement par telle maniere que a toutes heures et momens du jour et de la nuyt on puet veoir clerement en quel signe et degre les planetes sont, et estoilles sollennelles du ciel. Et est faicte si soubtilment ceste espere que nonobstant la multitude des roes, qui ne se pourroient nombrer bonnement sans deffaire l'instrument, tout le meuvement d'icelle est gouverne par ung seul contrepoiz, qui est grant merveille $[. . .]^{8}$.»

Cette description de l'horloge astronomique souligne la complexité de sa structure, gouvernée par des principes mathématiques : les cercles, la multiplication, l'interaction entre l'un et le multiple. Mézières remarque aussi que l'on ne pourrait pas compter les roues de l'horloge sans la défaire, une observation qui souligne l'importance du nombre dans la structure de l'horloge ainsi que l'air de mystère qui entourait sa fabrication. Et comme plusieurs autres auteurs de son époque, Mézières est impressionné par le fait que l'horloge fonctionne jour et nuit, "a toutes heures et momens". Ici il faut remarquer qu'à l'époque de Froissart les heures ne sont pas encore subdivisées en minutes : une horloge n'a qu'une aiguille et l'heure est la principale unité temporelle. Certains textes techniques font référence au moment, un terme précis qui désignait la quarantième partie d'une heure, ou une minute et demie. Mais en raison de l'imperfection des premières horloges, l'usage du moment comme mesure restait cantonné au plan théorique.

11 Jusqu'ici je n'ai parlé que des horloges publiques, pour la simple raison qu'au XIV e siècle les horloges privées étaient très rares; les rois de France en possédaient, mais les horloges portatives n'étaient pas communes avant le $\mathrm{XV}^{\mathrm{e}}$ siècle. Et il faut ajouter qu'au 
XIV e siècle ni les horloges privées ni les horloges publiques n'étaient fiables. Les premières horloges mécaniques, même la fameuse horloge du palais, avaient tendance à perdre au moins une heure par journée : Carlo Cipolla cite, par exemple, le vers

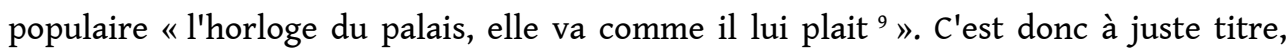
semble-t-il, que Jacques Le Goff affirme que " plus qu'outil de la vie quotidienne, elle est encore une merveille, un ornement, un jouet dont la ville s'enorgueillit. Elle appartient à la parure urbaine, au prestige plus qu'à l'utilité ${ }^{10}$ ».

L'horloge - et si nous poursuivons l'équation de Froissart, l'amant - est donc un objet fort admiré mais encore imparfait. Froissart souligne cependant les qualités de l'horloge théorique sans mentionner les défauts de l'horloge réelle.

«Car l'orloge est, au vrai considerer,

Un instrument tres bel et tres notable

Et s'est aussi plaisant et pourfitable,

Car nuit et jour les heures nous aprent

En l'absense meisme dou soleil.» (vv. 6-10)

13 Froissart semble reprendre le langage du décret (supposé) de Charles V, « luise le soleil ou non ", que nous avons déjà remarqué chez Philippe de Mézières. Après cet éloge de l'horloge, Froissart termine la partie introductive du poème, et l'énonciation en trois modes commence.

Les trois modes du discours

Je propose maintenant de démêler les trois modes du récit afin de les analyser individuellement, et ensuite de les réunir, tout en considérant la manière dont ce jeu triple mène à une réflexion sur l'allégorie amoureuse et fait partie d'un jeu plus large sur le nombre. Je vais d'abord regarder les passages écrits sur le mode technique, où Froissart démontre sa connaissance de la théorie de l'horlogerie. Ces parties du texte témoignent d'une préoccupation mathématique qui, à mon avis, caractérise la structure du poème entier.

15 Ces passages prennent le caractère d'un traité d'horlogerie en vers, avec des précisions mathématiques et un lexique spécialisé. Les vers 201 à 220 décrivent les deux premières roues et le foliot; les vers 347 à 366, le dyal et les clochettes; 599 à 614, la sonnerie ; et 927 à 948 , l'horloger. La description du dyal montre à quel point Froissart maîtrisait le vocabulaire spécifique de l'horlogerie. Ce mot est un néologisme dont Froissart nous donne l'une des premières attestations dans la langue française.

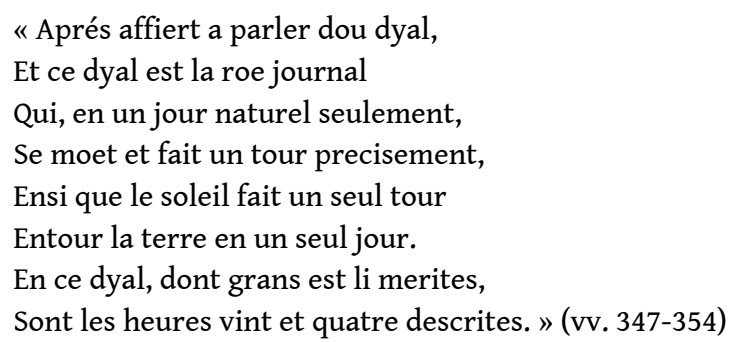

16 Cet extrait est intéressant pour sa considération du concept du naturel. Le dyal se trouve à l'intersection du naturel (le jour marqué par le mouvement apparent du soleil) et du mécanique (le jour marqué par les roues, les aiguilles et les clochettes). Ce jeu de la nature et de la technologie est également implicite dans le jeu qui structure le poème, celui du sentiment et de la machine. Mais c'est le monde mécanique qui domine le discours de ce mode technique, et la nature et l'homme y prennent une importance 
réduite. Avec la description de l'horloger dans le dernier passage écrit sur le mode technique, un élément humain est enfin introduit dans ce discours.

«Et pour ce que li orloges ne poet

Aler de soi, ne noient se moet,

Se il n'a qui le garde et qui en songne,

Pour ce li fault a sa propre besongne

Un orlogier avoir, qui tart et tempre

Diligamment l'aministre et attempre

Les plons relieve et met a leur devoir,

Et si les fait rieuleement mouvoir,

Et les roes amodere et ordonne,

Et de sonner l'ordenance lor donne.

Encores met li orlogiers a point

Le foliot, qui ne cesse point,

Le fuiselet et toutes les brochetes

Et la roe qui touce les clochetes.

Sont les heures, qui ens ou dyal sont,

De sonner tres certainne ordenance ont,

Mes que levee a point soit la destente.

Encores poet moult bien, selonc m'entente,

Li orlogiers, quant il en a loisir,

Toutes les fois qu'il li vient a plaisir,

Faire sonner les clochetes petites

Sans desrieuler les heures dessus dittes. » (vv. 927-948)

Dans le passage allégorique qui suit, Froissart dit que l'horloger représente Souvenir, mais il est aussi facile de voir les rapports entre l'horloger et la figure du poète. Celui qui soigne (songne) l'horloge amoureuse est aussi celui qui l'a songé, et qui lui donne « ordenance ». Ce passage est aussi unique parmi les extraits «techniques» du poème parce qu'il introduit l'idée du loisir et de la musique. L'horloge est souvent mentionnée ou illustrée comme instrument de musique. Dans le Roman de la Rose, Jean de Meung présente l'horloge non comme une machine à mesurer le temps, mais comme un instrument composé de clochettes. La musique, comme l'horloge, est fondée sur le concept de mesure : selon Christine de Pizan dans le Livre des fais et bonnes meurs du sage roy Charles $V$, «l'art de musique peut mesurer son ${ }^{11} »$. La musique apporte aussi le plaisir et le repos, comme le dit, entre autres, Eustache Deschamps dans l'Art de dictier. On peut remarquer que ce passage de Froissart est le seul dans lequel le poète mentionne le plaisir que l'on peut tirer de la structure mécanique de l'horloge. Cet emploi de la machine serait analogue à l'amour physique (l'emploi du corps pour le plaisir) et à la poésie (la création d'une « musique » des paroles).

Comme je viens de le dire, ces passages techniques sont caractérisés par une précision conceptuelle et lexicale. Paul Zumthor a déjà remarqué les ressemblances entre la description de Froissart et celle d'un traité technique anonyme, datant du milieu ou de la seconde moitié du XIV ${ }^{\mathrm{e}}$ siècle ${ }^{12}$. Le traité, caractérisé dans son incipit comme « ung petit traictie pour faire horoleiges en plusieurs manieres selon l'ymagination du fayseur » (275) - ainsi que la Recapitulatio brevis en latin qui le suit - décrit le même genre d'horloge que celui de l'Orloge amoureus. La précision mathématique de ce traité pratique a déjà été notée par Paul Zumthor : en effet, il s'agit d'un système sophistiqué dans lequel l'horloge devient symbolique de l'union du pair et de l'impair. C'est précisément de cette union que dépend l'horloge mécanique, dont l'échappement à roue de rencontre fonctionne en faisant s'articuler une roue avec un nombre pair de 
dents et une autre avec un nombre impair de dents : « Et est assavoir que en ladite roue $\mathrm{du}$ foliot doivent les dens estre toujours non per affin que au rencontre, quant elle fiert a une des dens, elle puisse eschapper par entre les aultres dens... » (276). Ainsi, dans le traité anonyme, nous voyons que la roue centrale a un nombre pair de dents; le foliot, un nombre impair ; la roue du soleil, pair ; la roue de la lune, impair.

Bien sûr, l'auteur du traité d'horlogerie emploie le langage spécialisé de son métier ; il est donc intéressant de regarder les ressemblances entre son lexique et le langage de Froissart dans l'Orloge amoureus. Le mot dyal, dont j'ai déjà signalé l'usage chez Froissart, est expliqué par l'horloger anonyme : « il est dit dyal pour ce qu'il ne fait en ung jour que ung tour " (279). D'après Paul Zumthor, la définition du terme dans un ouvrage pourtant spécialisé atteste sa nouveauté. Cet exemple ne fait que confirmer la sophistication technique de la description de l'horloge que nous donne Froissart.

Mais la nouveauté chez Froissart est restreinte à son mode technique et à la manière dont le poète lie sa matière "moderne " aux conventions de l'allégorie amoureuse. Car dans les passages du mode allégorique, Froissart attribue une signification à chaque partie de l'horloge de façon très explicite, voire plate. Nous pouvons prendre comme exemple la description de la première roue aux vers 99 à 108 .

«Or voeil parler de l'estat de l'orloge.

La premerainne roe qui y loge,

Celle est la mere et li commencemens

Qui fait mouvoir les aultres mouvemens

Dont l'orloge a ordenance et maniere.

Pour ce poet bien ceste roe premiere

Segnefier tres couvignablement

Le vrai desir qui le coer d'omme esprent ;

Car desir est la premiere racine

Qui en amer par amours l'enracine. »

D'abord, il semble qu'il n'y ait rien de subtil ici : le poète nous déclare sans ambiguïté que la «premerainne roe» signifie Désir. Il fait de même avec les contrepoids, qui représentent Beauté et Plaisance; la seconde roue, Attemprance; le foliot, Paours; le dial, Douç Penser; et cætera. Il va jusqu'à attribuer une signification allégorique à chacune des vingt-quatre "broquetes». Françoise Paheau a remarqué que les désignations de ces personnages allégoriques font preuve d'une attitude «tout à fait favorable » envers l'horloge, car il n'y a pas de personnages négatifs tels Malebouche ou Dangier ${ }^{13}$. Pourtant, Paour est présent dans l'allégorie. De plus, Malebouche et Dangier n'auraient aucune place dans le système de l'Orloge amoureus : comme l'horloge figure le cœur de l'amant - c'est-à-dire son univers interne -, on ne s'attendrait pas à trouver des obstacles externes comme Malebouche ou Dangier, qui représentent les attitudes des jaloux ou de la dame.

Revenons maintenant aux derniers passages cités. Dans l'explication de la première roue il est question de mouvement, d'ordenance et de mesure, tout comme dans le passage qui décrit l'horloger qui «diligamment l'aministre et attempre» (v. 932). «Attempre » est un mot-clé : il n'est donc pas surprenant que «Attemprance » figure parmi les personnages évoqués dans le discours allégorique. En effet, dans l'univers iconographique du Moyen Âge tardif, Temperantia est souvent associée à l'horloge.

Même avant l'époque de Froissart, il est commun de voir des descriptions ou des images qui représentent l'univers comme une horloge, et qui représentent Dieu en horloger avec son compas. Pourtant, l'association entre «Attemprance» et une partie de 
l'horloge semble trouver son origine chez Froissart. C'est une association que Nicole Oresme reprend dans son Livre du ciel et du monde (1377) et qui se développe encore plus dans l'Epistre Othéa de Christine de Pizan (1400-01). Dans ce texte, composé lui aussi de trois « modes » - texte, glose et allégorie -, «Attrempance » est la seconde figure à être commentée, juste après sa "seur germaine », la déesse Othéa. Ici c'est tout le corps humain, et non seulement le cœur, qui peut être représenté par l'horloge : « et pour ce que nostre corps humain est composé de diverses choses et doit estre attrempé selon raison, peut etre figuré à l'orloge qui a plusieurs roes et mesures; et toutefoiz ne vault rien l'or[lo]ge, s'il n'est attrempé, semblablement non fait nostre corps humain, se attrempance ne l'ordonne ${ }^{14}$ ». "Attrempance » pèse et mesure l'activité humaine comme un horloger règle l'activité d'une horloge : «Car s'elle n'en faisoit le pois,/ Tout ne te vauldroit pas un pois » (vv. 25-26, p. 203). Ce lien entre la mesure du temps et la mesure dans l'activité humaine est souligné dans le programme iconographique des enluminures qui accompagnent souvent l'Epistre Othea : cette série d'enluminures inclut l'image d'« Attemprance » qui règle le mécanisme d'une horloge. Cette figure ressemble beaucoup à celle de Sapientia qui apparaît dans certains manuscrits de l'Horloge de Sapience d'Henri Suso. Et Dohrn-Van Rossum cite aussi un groupe d'enluminures du Xv siècle qui figurent Temperantia d'une manière particulière : elle est entourée par les grands monuments de la technologie moderne - des lunettes, une bride, des éperons, un moulin à vent, et sur sa tête, une horloge. Nous voyons alors que l'association entre la mesure mécanique et la mesure comme vertu, faite par Froissart, a une longue postérité dans la littérature et l'iconographie médiévales.

Naturellement, chez Froissart, ce concept de la mesure s'applique aussi bien au comportement de l'amant qu'à la mécanique de l'horloge. Dans l'explication allégorique de la roue de la sonnerie et de son contrepoids, Froissart fait l'éloge de la mesure.

«Et pour ce qu'il [le vrai amant] aussi ne passe point

La mesure de raison, fors a point,

Il li convient, par bonne entention,

Mettre en son coer toute discretion. » (vv. 649-652)

Ce souci de l'ordenance relie les trois modes du texte, et la structure triple de l'ouvrage a pour effet d'imposer l'ordre de l'horloge mécanique sur le désordre fondamental de l'amour. Dans les passages écrits dans le mode direct, l'auteur s'engage souvent dans une discussion du problème de l'ordre en relation avec une réflexion sur la composition de l'ouvrage.

«Si vous suppli, ma dame, qu'en ceste oevre

Vous m'escusés, se rudement g'i oevre.

Mes pour le mieulz a mon pooir m'ordonne,

Selonk le droit que li orloges donne,

A qui me sui proprement comparés. » (vv. 335-39)

Ainsi Froissart réitère la comparaison avec laquelle il a ouvert son texte, cette fois dans le contexte de l'ordenance mécanique. "Le droit que li orloges donne" est une ordonnance qui s'impose sur, et dans, le corps de l'amant-poète. La comparaison de base entre le poète et l'horloge nécessite une assimilation, une incorporation de la machine chez l'amant. Le sujet amoureux devient une espèce d'homme bionique - la machine remplace un de ses organes vitaux, et il se comporte «A la façon proprement de l'orloge,/ Dont Amours fait de mon coer chambre et loge » (vv. 49-50). À la fin du poème, ce lien entre l'homme et la machine est de nouveau souligné. 
«Car je sui la chambre et la maison

Ou mis est li orloges amoureus,

Sui de mouvoir telement curïeus

Que n'ai aillours entente, soing et cure,

Ne Nature riens el ne me procure

Fors que tout dis mouvoir sans arrester.

$\mathrm{Ne}$ je ne puis une heure en paix ester,

Meïsmement quant je sommeille et dors. » (vv. 1152-59) maladie. par l'intellect de l'amant.

Esperance et imagination

Nature l'aide donc à imiter la machine, car la perturbation de l'amant est constante, tout comme le mouvement de l'horloge. Ce passage peut nous rappeler la description aux vers 9 et 10 de l'horloge qui «nuit et jour les heures nous aprent/ En l'absense meisme dou soleil»; ici l'analogie entre l'horloge royale, l'horloge de Froissart et l'amant est claire. Même les mutations qui ont lieu dans l'âme de l'amant s'expliquent par la structure et le langage de l'horloge.

"Quanque je voi, une heure bien me plest,

Et puis tantos ce que voi me desplest.

Une heure voeil je estre en compagnie

L'autre le fui, avoir ne le voeil mie. » (vv. 735-738)

Comme nous l'avons déjà mentionné, l'invention de l'horloge mécanique provoque un changement profond dans le sens du mot " heure». La régularité relative des heures modernes était soulignée par les clochettes qui sonnaient à des intervalles réguliers. Et si l'horloge marque le passage des heures avec la musique, le poète marque les mouvements de son âme avec la poésie. Dans le texte de Froissart, la sonnerie - et, par analogie, sa propre production poétique - évoque en même temps la mélodie et la

« En ce frefel et en celle rihote

Fai maint souspir, maint plaint et mainte note

Qui ne sont pas de sons melodieus,

Mes attemprés de chans maladïeus. » (vv. 857-860)

Le rôle d'« Attemprance » est d'intercaler le positif et le négatif, d'attemprer l'harmonie ( "les sons melodieus ») avec la discorde («les chans maladieus») afin de trouver un équilibre entre les deux. Le poème en est le résultat. Cependant, cette "sonnerie " poétique doit forcément dépasser la sonnerie mécanique de l'horloge. La machine, même si elle remplace le cœur de l'amant, est insuffisante en soi et doit être complétée

« Car se le vrai amant ne concevoit

En sa pensee, et aussi s'il n'avoit

De parvenir, a la conclusion,

A son entente et a ce qu'il desire,

Les heures amoureuses, au voir dire,

Ne poroient sonner souffissament. » (vv. 477-483)

Cette déclaration pourrait nous aider à comprendre la structure du poème : le mode « direct » vient toujours « à la conclusion » après les deux autres parce que le poète doit supplémenter la mécanique technique et allégorique avec sa propre pensée et sa propre imagination afin de produire un texte qui « sonne souffissament ».

31 La jonction des trois modes peut nous sembler parfois trop évidente, surtout en ce qui concerne les liens explicites entre les modes technique et allégorique. En revanche, la correspondance entre la mécanique et l'allégorique n'est jamais aussi stable qu'elle ne 
le semble : tout en établissant ces analogies, le poète attire notre attention sur le fait qu'il s'agit, en effet, d'un jeu. La première roue poet bien représenter le désir ; ainsi Froissart indique que sa configuration des figures conventionnelles de l'allégorie amoureuse n'est qu'une configuration parmi une multitude de possibilités. Cette expression de potentialité plutôt que de vérité absolue - poet bien - crée un espace entre les niveaux technique, allégorique et direct du texte, qui permet un glissement et qui constitue une reconnaissance de l'artifice de la poésie amoureuse. Si l'horloge du discours technique est liée à l'amant du discours direct, c'est seulement grâce à l'intervention du discours allégorique; mais admettre que le rapport entre le niveau technique et le niveau direct n'est qu'un rapport possible met en doute la validité, voire la raison d'être, du niveau intermédiaire. Douglas Kelly constate que la sublimation de l'auteur dans une figure comme l'horloge mène à un effet de "dépersonnalisation » ${ }^{15}$. À mon avis, l'aveu de l'artifice implicite dans les mots poet bien suggère que ce n'est pas le recours à une machine pour figurer un sentiment, mais plutôt le recours aux figures allégoriques, qui effectue cette dépersonnalisation.

Je lis donc la division du texte en trois modes distincts comme une mise à nu du processus allégorisant. Le poème s'approche par étapes de l'expérience amoureuse, passant de la machine à l'homme à travers les conventions allégoriques. Comme le traité d'horlogerie, le poème de Froissart expose le mécanisme de la machine qu'il décrit: dans ce cas, la "machine» en question est l'écriture amoureuse. Le texte commence par une espèce de défi - je me puis bien comparer a l'orloge - et le reste du poème constitue une preuve de ce théorème. Froissart montre comment un poète poet bien choisir n'importe quel objet (Froissart tire son exemple de l'actualité, la nouvelle horloge du palais), attribuer des significations allégoriques aux parties de cet objet, et traduire ce système allégorique en un appel direct à une dame ${ }^{16}$.

Le thème de l'horloge entre texte et image

Son analogie principale rapproche l'Orloge amoureus de quelques autres textes du XIV siècle qui emploient la figure de l'horloge. Cependant, la complexité du jeu entre amant, personnification allégorique et horloge sépare le texte de Froissart des autres. Une comparaison avec deux autres textes évoquant l'horloge peut permettre de saisir l'originalité de Froissart : la Divine Comédie de Dante, et l'un des textes plus populaires du Moyen Âge tardif, l'Horologium sapientiae (ou Horloge de Sapience) d'Henri Suso.

Chez Dante, le temps, et la capacité d'en jouir, font partie de son système élaboré de punitions et de récompenses. Le vestibule des enfers, par exemple, existe dans une "aura senza tempo" (Inferno III, v. 29), alors que l'auteur emploie l'analogie de l'horloge - une description souvent citée par les historiens de l'horloge - dans le Paradiso chant $\mathrm{X}$, vers 139-148. Ici le pèlerin-narrateur fait une comparaison entre une horloge, dont la sonnerie est décrite avec délicatesse, et la « gloriosa rota » d'âmes qu'il voit dans la sphère du soleil.

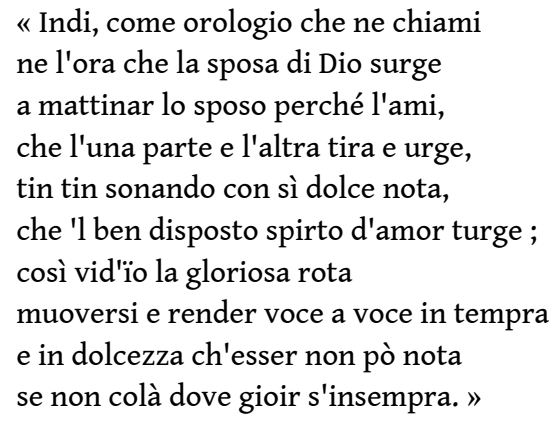


«Puis, comme une horloge qui nous appelle,

à l'heure où se lève l'épouse de Dieu

pour faire matine à son époux afin qu'il l'aime,

tandis qu'une pièce tire et pousse l'autre,

en sonnant et tintant en notes si douces

que l'esprit préparé se gonfle d'amour ;

je vis ainsi la roue glorieuse

se mouvoir et accorder ses voix

dans une douceur qu'on ne peut connaître

sinon là où la joie s'éternise. »

Comme dans le Roman de la Rose, Dante souligne l'aspect sonore de l'horloge : le doux tin tin de l'horloge est assimilé au son des voix célestes. Il existe aussi un rapport plus formel entre les roues de l'horloge et la "gloriosa rota » composée d'âmes saintes, ainsi qu'une évocation du mouvement - un mot qui, comme nous le savons, est d'une grande importance dans l'horlogerie. Cependant, ces comparaisons restent plus ou moins implicites, et l'accent est mis plus sur la fonction de l'horloge - elle appelle «la sposa di Dio » aux prières - que sur sa structure.

Cette même indifférence aux mécanismes de l'horloge caractérise aussi l'Horologium sapientiae d'Henri Suso, un ouvrage qui donne, en principe, une importance majeure à la figure de l'horloge par rapport à la Divine Comédie de Dante. Le texte de l'Horologium sapientiae nous est parvenu dans à peu près cinq cents manuscrits des $\mathrm{XIV}^{\mathrm{e}}$ et $\mathrm{XV}^{\mathrm{e}}$ siècles. Il existe des traductions médiévales en plusieurs langues vulgaires; la traduction française date de 1389. En abordant ce texte, le titre nous donne l'impression que l'image de l'horloge y sera centrale. Mais en fait, la figure de l'horloge n'est pas indispensable au texte, et à part le titre et une brève mention dans le prologue, l'horloge est tout à fait absente. Comme Froissart, Suso établit la métaphore de l'horloge dans son prologue :

«Unde et praesens opusculum in visione quadam sub cuiusdam horologii pulcherimmi rosis speciosissimis decorati et cymbalorum bene sonantium et suavem ac caelestem sonum reddentium cunctorumque corda sursum movientum varietate perornati figura dignata est ostendere clementia salvatoris ${ }^{17}$.»

$\mathrm{Ou}$, comme le dit la traduction française, Sapience «lui monstra ung horloge ou ologe de tresbelle et de tresnoble forme dont les roes estoient excellentes et les cloches doulcement sonnans et par la diverse et subtille façon de lui, tout cuer humain semerveilloit et esjoissoit en regardant ycelui ${ }^{18} »$. Cette description de l'horloge rappelle celle de Dante dans son insistance sur les «cymbalorum bene sonantium » et l'appel à la prière. Suso ajoute le détail de la décoration de l'horloge avec des roses - un détail apparemment mal compris par le traducteur français, qui parle de «roes » au lieu de "roses ». Après le prologue, l'Horologium sapientiae est divisé en vingt-quatre chapitres, ce qui le rapproche en même temps du livre d'heures (et, par extension, de l'ancien système canonial marquant le passage du temps) et de l'horloge. Mais ce lien avec l'horloge n'est jamais mentionné, et il semble que l'horloge ne figure pas dans le reste du texte : ayant appelé le lecteur à la contemplation de Dieu, ayant ainsi joué son rôle, elle est mise de côté et oubliée. Malgré son absence de la majeure partie du texte, la figure de l'horloge semble avoir captivé l'imagination de plusieurs enlumineurs qui ont illustré les manuscrits de l'Horologium et de ses traductions vulgaires. La plus connue de ces enluminures est celle du manuscrit de la Bibliothèque Royale de Bruxelles (MS IV.111, fol. 13v.), qui date du milieu du $\mathrm{Xv}^{\mathrm{e}}$ siècle et qui met en scène «Sapience» et son «Disciple» entourés d'une variété d'horloges et d'instruments 
astronomiques. Le manuscrit inclut une table des enluminures ou «declaration des hystoires ", ajoutée pendant la deuxième moitié du xve siècle, qui décrit cette image de la façon suivante :

«Pource que comme ie dit pou davant la divine sapience est toujours soigneuse du sauvement de toute humaine creature, elle rallume les étains, elle rechauffe les refroidis et resuscite les endormis. En ce signifiant est nommé ce livre horloge de sapience. Et en cette figure est dame sapience comme ordonnant et gouvernant une horloge et ung refueil disent en son de plusieurs clochettes ante secula qui deus est tempora homo factus est in maria, lequel verset est approprie convenablement à notre seigneur ihucrist qui est l'eternelle sapience et filz de dieu le pere et a prins char humaine et nasqui en la vierge marie. Et à ses piez est le disciple contemplant le maintien de sa maistresse ${ }^{19}$.

Une enluminure dans un manuscrit de Vienne (NB cod. 2574, fol. 24r.), très proche de celle-ci, montre la «Sapience » qui ajuste une horloge. Ces images, surtout celle du manuscrit de Bruxelles, ont eu une valeur importante pour les historiens de l'horlogerie ; comme le poème de Froissart, elles fournissent une représentation des horloges de l'époque qui est, de toute évidence, assez exacte. Malheureusement, le texte de l'Orloge amoureus dans le seul manuscrit qui nous le transmet n'est orné d'aucune enluminure. Cependant, le texte nous donne une très bonne idée de ce qu'était une horloge mécanique en 1368. L'assimilation de la machine au corps de l'amant est réalisée par un jeu subtil et non sans intérêt qui expose et, d'une certaine manière, déconstruit le travail que fait le créateur d'une allégorie amoureuse. C'est précisément à cause de cette réflexion sur l'instabilité du texte allégorique que je ne peux pas adhérer à l'affirmation de Huizinga selon laquelle l'Orloge amoureus est un poème sans originalité et sans intérêt.

\section{NOTES}

1. Une première version de cet article a été présentée au séminaire de $\mathrm{M}^{\mathrm{me}} \mathrm{le}$ professeur Jacqueline Cerquiglini-Toulet, « Le Nombre de la poésie », en mai 2004. Je voudrais remercier Mme Cerquiglini-Toulet et Mlle Delphine Louis pour leurs commentaires et leurs corrections. Les fautes qui restent sont miennes.

2. J. Huizinga, L'Automne du Moyen Âge, Paris 2002, p. 319.

3. BnF ms. fr. 176, fo $13 \mathrm{v}^{\circ}$. Cité dans G. DoHRn-van Rossum, History of the Hour: Clocks and Modern Temporal Orders, Chicago, 1996, p. 405, note 4.

4. Jacques Le Goff, «Au Moyen Âge : Temps de l'Église et temps du marchand » dans Pour un autre Moyen Âge: Temps, travail et culture en occident, Paris, 1977, p. 46-65.

5. Ibid., p. 218.

6. C. de Pizan, Livre des fais et bonnes meurs du sage roy Charles V, S. Solente éd., Paris, 1936, p. 42.

7. J. Froissart, Le Paradis d'Amour/L'Orloge amoureus, P. Dembowski éd., Genève, 1986, p. 11. Toutes les citations de Froissart sont extraites de cette édition.

8. Ph. de Mézieres, Le Songe du vieil pélerin, G. W. Coopland éd., 2 t., Cambridge, 1969, p. 606. 
9. C. Cipolla, Tecnica, società e cultura : Alle origini della supremazia tecnologica dell'Europa (XIV-XVII secolo), Bologne, 1967, p. 27.

10. J. Le Goff, loc. cit., p. 75.

11. C. DE PIZAN, op. cit., p. 45.

12. BAV, ms. lat. 3127. Publié et commenté par P. Zumthor, « Un traité français d'horlogerie du xiv ${ }^{\mathrm{e}}$ s. », Zeitschrift für romanische Philologie, 73, 1957, p. 274-287.

13. F. Paheau, «Scientific Allusions and Intertextuality in Jean Froissart's L'Orloge amoureus ", Journal of Medieval and Renaissance Studies, 20, 1990, p. 256.

14. C. de Pizan, Epistre Othea, G. Parussa éd., Genève, 1999, p. 202.

15. D. Kelly, «The Genius of the Patron : The Prince, the Poet, and Fourteenth-Century Invention », dans R. BARTON PALMER éd., Chaucer's French Contemporaries : The Poetry/ Poetics of Self and Tradition, New York 1999, p. 11.

16. Il ne serait pas sans intérêt de noter ici la ressemblance avec un autre texte allégorique construit à partir d'une analogie entre un personnage et un objet : il s'agit du Dit de la Harpe de G. de Machaut. Les premiers vers de ce dit - « Je puis trop bien ma dame comparer/a la harpe... » (BnF, ms. fr. 1584, fo 174r.) - ont sans doute eu une influence directe sur l'Orloge amoureus.

17. H. Suso, Heinrich Seuses Horologium Sapientiae, D. PlanzER éd., Fribourg 1977, p. 364-65.

18. Cité dans E. P. Spencer, «L'Horloge de Sapience, Bruxelles, Bibliothèque Royale, MS. IV.111 », Scriptorium, 17, 1963, p. 282-283.

19. fo 3 ; cité dans E. P. Spencer, op. cit., p. 283.

\section{RÉSUMÉS}

L'Orloge amoureus de Jean Froissart, un poème narratif rédigé vers 1368, se construit à partir d'une comparaison simple : l'amant est comme une horloge, ou plus précisément, l'amant peut bien être comparé à l'horloge. Dans cet article nous proposons de résumer les innovations de l'horlogerie au XIV ${ }^{\mathrm{e}}$ siècle et de situer le poème de Froissart dans le contexte d'autres ouvrages contemporains traitant de l'horloge ou de l'horlogerie. L'élaboration de l'Orloge amoureus en trois «modes» de narration lui donne à la fois le caractère d'un traité de l'horlogerie, d'une description allégorique de la psychologie de l'amant, et d'un appel direct à la dame aimée. Alors que la composition du texte à partir d'un modèle mécanique met en relief les concepts fondamentaux de l'ordre et de la mesure, l'instabilité inhérente au rapport entre les trois modes expose et déconstruit le travail que fait le créateur d'une allégorie amoureuse.

Clockmaking and the Mechanics of Allegory in Jean Froissart's Orloge amoureus. Jean Froissart's Orloge amoureus, a narrative poem composed around 1368, is constructed upon a seemingly simple analogy : the lover is like a clock, or more precisely, a lover could well be compared to a clock. The present study proposes to summarize the innovations of fourteenth-century clockmaking and to situate Froissart's poem within the context of other late medieval texts employing the figure of the clock. The text's elaboration in three narrative "modes " make of it at once a treatise on clockmaking, an allegory of the psychology of love, and a direct appeal to the beloved lady. While the text's dependence on a mechanical model underlines the concepts of order and measure, the 
inherent instability of the relationship between the three modes lays bare and deconstructs the mechanisms of conventional love-allegory.

INDEX

Mots-clés : Jean Froissart, Orloge amoureus, horlogerie, allégorie

\section{AUTEUR}

JULIE SINGER

Department of Romance Studies, 205 Language Center, Box 90257, Duke University, Durham, NC 27708, USA 